\title{
On the problem of energy efficiency of multi-hop vs one-hop routing in Wireless Sensor Networks
}

\author{
Szymon Fedor and Martin Collier \\ Research Institute for Networks and Communications Engineering (RINCE), \\ Dublin City University, Dublin 9, Ireland, \\ Email: $\{$ fedor.szymon, collierm $\} @$ eeng.dcu.ie
}

\begin{abstract}
The hop distance strategy in Wireless Sensor Networks (WSNs) has a major impact on energy consumption of each sensor mote. Long-hop routing minimizes reception cost. However, a substantial power demand is incurred for long distance transmission. Since the transceiver is the major source of power consumption in the node, optimizing the routing for hop length can extend significantly the lifetime of the network. This paper explores when multi-hop routing is more energy efficient than direct transmission to the sink and the conditions for which the two-hop strategy is optimal. Experimental evidence is provided in to support of these conclusions. The tests showed that the superiority of the multi-hop scheme depends on the source-sink distance and reception cost. They also demonstrated that the twohop strategy is most energy efficient when the relay is at the midpoint of the total transmission radius. Our results may be used in existing routing protocols to select optimal relays or to determine whether it is better to send packets directly to the base station or through intermediate nodes.
\end{abstract}

\section{Introduction}

A fundamental design choice in Wireless Sensor Networks (WSNs) routing concerns whether packets should be sent over many short hops or over a smaller number of longer hops. Recently this debate has drawn significant research attention $[7,3,4,5]$.

WSNs have unique properties among ad-hoc networks. Their main difference comes from the fact that they are composed of miniature devices whose energy resources are strictly limited. This is why the network lifetime should be a major concern in designing communication protocols, especially as transmission may constitute the primary source of energy consumption in the node. Therefore, although many metrics may be used in the comparative evaluation of short-hop versus long-hop routing in ad-hoc networks (e.g. interference, mobility of the nodes, delay etc.), paramount among these is energy efficiency.

The debate over the number of required hops comes from the fact that each strategy (long-hop and short-hop routing) has its own advantages. Routing over many short hops minimizes the transmission energy which increases with the communication distance. However, sending packets over long distance relays reduces the reception cost (as the number of nodes involved in data routing decreases).

- We consider WSNs of large density with many nodes located between source and the sink. Such topology can be encountered with WSNs used for monitoring the environment, offices, systems or industrial sites.

- To compare the energy efficiency of different schemes, we use the Minimum Energy Routing (MER) metric which incorporates the total energy consumed on a path.

\section{Related work}

The issue of routing packets over long hops or short hops has been raised by many authors in recent years and their conclusions are varied depending on the approach taken and criteria considered.

Yin et al. [7] presented a two-level strategy for topology control in wireless sensor networks. One of the methods of transmission energy minimization consists of decreasing the transmission range of each node. According to the authors, this scheme will reduce the overall power consumption of the network, as a route with many short hops is generally more energy-efficient than one with a few long hops.

Haengi specified many reasons why long-hop routing is more advantageous $[3,4,5]$. One of them is the power efficiency of such a strategy. The author claimed that although the transmitted energy drops significantly with distance, the reduction of radiated power does not yield a decrease in the 
total energy consumption. Haengi's experiments showed that routing as far as possible is a very competitive strategy in many cases.

The models of energy consumption used in the above studies do not accurately reflect the performance of practical WSN nodes, because they assume a constant energy cost (sometimes zero) per bit during transmission and reception. We investigate a more realistic model of the power required to communicate over a given distance, taking into consideration the power specifications of commercially available nodes. We use this to compare the total energy consumed using the long-hop and short-hop strategies and to identify the situations when the short-hop strategy gives better performance.

\section{Problem formulation}

\subsection{Applications considered}

Numerous possible applications have been proposed for WSNs. They can be used for monitoring the environment, habitat, offices, systems, buildings, and industrial sites. They can help rescue teams gather information about dangerous situations or people in need of assistance. They can be included as part of a patients monitoring system in hospitals. There are many others situations where WSNs could be employed. However in our study we wish to focus on the first mentioned group of applications. Buildings, sites, structural, habitat and environmental monitoring represent a very broad class of sensor network usage with enormous potential benefits for scientific communities and industry. Recent deployments of WSN, being one of the first robust and successful, were realized for the environment or habitat monitoring and proved vast utility of such systems in those areas. These applications will dictate the type of network topology we have to examine.

\subsection{Network topology}

In systems monitoring the environment or offices, WSNs are generally deployed over large areas with hundreds or thousands of nodes. Sensors collect the information periodically and transmit it to the one location called the base station. This communication scheme limits the number of established links, because for every node the final destination is always the base station. As the network is very dense, we can assume that there are a large number of relays aligned along the path joining the source to the destination. In previously mentioned applications, nodes are immobilized and they send a report about monitored parameters periodically to the base station. The network protocol overhead is very small because once the communication architecture is established, it can last for a long time (depending on the rout- ing strategy undertaken). Its energy efficiency may be evaluated using many metrics.

\subsection{Energy efficiency}

Among many WSN routing protocols we can find those which try to send packets over the longest possible hops to reduce the overall "linkload" between nodes. Others may implement the short-hop strategy because of the significant signal attenuation. Each of the strategies can be advantageous and hence we need a metric to compare energy efficiency of the long-hop and short-hop scheme. We can use one of three criteria of evaluation. The first is called Minimum Transmission Energy (MTE), and consists of comparing only the energy used for the transmission by every node. Another is the Minimum Energy Routing (MER) which incorporates the total energy consumed on a path. The final, called Maximum Network Lifetime (MNL) considers the lifetime of the network which represents the time until the first depletion of a node's battery. We want to select the MER metric for the following reasons.

- The specifications of some WSNs transceivers show that reception cost can be even higher than transmission cost (figure 1).

- MNL seeks to maximize the lifetime of the first node to fail. However, in the applications considered here, the system may survive the failure of portion of the nodes.

- The strategy of seeking to maximize network lifetime may be inappropriate in WSNs since its complexity implies a considerable power budget.

We also think that in many cases the strategy of balancing the energy remaining on the nodes may be inappropriate to undertake because it requires a significant protocol overhead and in case of not uniform distribution of energy resources of the nodes, the cost of prolonging the network lifetime can be unreasonable. In the next section we specify the problem we want to solve for a given WSN application and using a described energy efficiency metric.

\subsection{Questions to address}

We consider very dense WSNs with many potential relays to the base station. In such cases, a short-hop routing scheme is never most advantageous using the MER metric. This is mainly caused by the significant energy consumption of each reception in existing WSNs (see figure 1) which may be even higher than when the node is transmitting. As a consequence we would like to find out whether and 


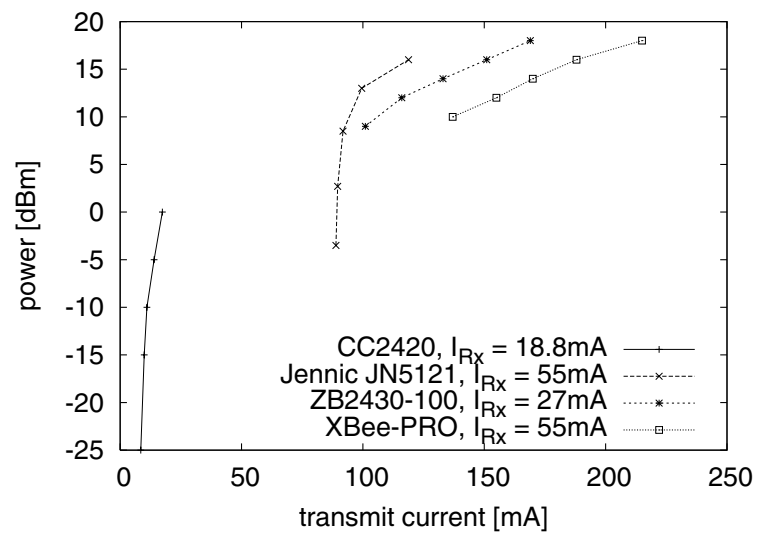

Figure 1. Current consumption in transmission (for different RF power levels) and reception $\left(I_{R x}\right)$ of existing WSN modules: CC2420 (voltage supply 3V), XBee-PRO (3.3V), Jennic JN5121-000-M02 (3V), ZB2430100 (3.3V).

when the long-hop strategy is optimal using the MER metric. With this scheme a large portion of nodes can communicate directly to the base station since the outdoor transmission range can attain $1200 \mathrm{~m}$ [2]. Hence we consider when the nodes should relay packets through other sensors or send it to the final destination. We want to know also which twohop topology is optimal. We examine those questions first with an analytical study.

\section{Analytical study}

We approach the multi-hop energy efficiency problem in the following way. First, we describe the wave propagation model used in our study. Next, we explain why we should compare the two-hop transmission with direct communication in order to deduce when multi-hop routing is advantageous using the MER metric. Then, we study the total power radiated using both schemes: single and multihop sending. In the next step, we link the power radiated by the node to the energy drained form the battery. Finally, we conclude when each of the strategies considered should be applied.

\subsection{Propagation model}

In our study we use the log-distance path loss model [6]. The power received by a node distant of $d$ meters from the sender can be expressed as follows:

$$
P(d)=P_{0} \times\left(\frac{d_{0}}{d}\right)^{\alpha}
$$

where $P_{0}$ represents the power of the signal received at distance $d_{0}$ from the source and $\alpha$ is the path loss exponent. The value of $\alpha$ depends on the specific propagation environment and experiments have shown that it usually takes a value between 2 and $5[1,6]$. We use eq. (1) to express the minimum power required to communicate over a given distance and we compare the two routing strategies.

\subsection{Multi-hop vs Single-hop problem refor- mulation}

When the nodes are communicating with the minimum power necessary to reach the destination, if we consider only the total power transmitted over the path, then the short-hop strategy would be the most energy efficient. This is caused by the signal attenuation which is proportional to the power function (eq. (1)). However, since the reception cost should not be neglected, there is a minimum range of source-destination distance for which direct communication is an optimal alternative. This is due to the fact that the savings in transmission power by the multi-hop scheme does not compensate for the resulting additional reception energy cost. If we enlarge the distance between source and base station, there is a bound for which the two-hop routing becomes more advantageous. If the transmission distance increases, the 3-hop communication will become optimal and so forth. Therefore if we wish to know which routing strategy is more energy efficient for a given topology then we need to compare the energy consumption of single-hop and two-hop transmission. Figure 2 represents the two-hop

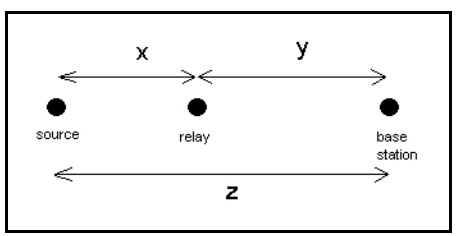

Figure 2. One-hop and direct transmission.

and direct transmissions. Every node is transmitting with the minimum power required to guarantee the signal at the receiver is above the sensitivity level $P_{m}$. For each link we can therefore write:

$P_{m}=P_{x} \times\left(\frac{d_{0}}{x}\right)^{\alpha}=P_{y} \times\left(\frac{d_{0}}{y}\right)^{\alpha}=P_{z} \times\left(\frac{d_{0}}{z}\right)^{\alpha}$

We can now compare when two-hop routing is more energy efficient:

$$
P_{x}+P_{y}+P_{r}<P_{z}
$$

where $P_{r}$ represents power drained at the reception from the relay. If we take $P_{x}, P_{y}$ and $P_{z}$ from the eq. (2), we get

$P_{m} \times\left(\frac{x}{d_{0}}\right)^{\alpha}+P_{m} \times\left(\frac{y}{d_{0}}\right)^{\alpha}+P_{r}<P_{m} \times\left(\frac{z}{d_{0}}\right)^{\alpha}$ 


\begin{tabular}{|c|c|c|}
\hline$\alpha$ & $\begin{array}{c}\text { Source-relay distance when } \\
\text { two-hop routing is energy optimal }\end{array}$ & $\begin{array}{c}\text { reception energy } \\
\text { condition }\end{array}$ \\
\hline 2 & $x \epsilon\left(\frac{z}{2}-\frac{\sqrt{z^{2}-2 t^{2}}}{2} ; \frac{z}{2}+\frac{\sqrt{z^{2}-2 t^{2}}}{2}\right)$ & $z>t \sqrt{2}$ \\
3 & $x \epsilon\left(\frac{z}{2}-\frac{\sqrt{3 z\left(3 z^{3}-4 t^{3}\right)}}{6 z} ; \frac{z}{2}+\frac{\sqrt{3 z\left(3 z^{3}-4 t^{3}\right)}}{6 z}\right)$ & $z>t \sqrt[3]{\frac{4}{3}}$ \\
4 & $x \epsilon\left(\frac{z}{2}-\sqrt{\frac{-3 / 2 \times z^{2}+\sqrt{4 z^{4}-2 t^{4}}}{2}} ; \frac{z}{2}-\sqrt{\frac{-3 / 2 \times z^{2}+\sqrt{4 z^{4}-2 t^{4}}}{2}}\right)$ & $z>t \sqrt[4]{\frac{8}{7}}$ \\
5 & $x \epsilon\left(\frac{z}{2}-\sqrt{\frac{-1 / 2 \times z^{2}+\sqrt{z^{4}-4 / 5 \times t^{5} / z}}{2}} ; \frac{z}{2}+\sqrt{\frac{-1 / 2 \times z^{2}+\sqrt{z^{4}-4 / 5 \times t^{5} / z}}{2}}\right)$ & $z>t \sqrt[5]{\frac{16}{15}}$ \\
\hline
\end{tabular}

Table 1. Multi-hop energy efficiency advantage for different values of $\alpha$.

The energy cost of the reception can be equivalent to transmitting over a distant $t$. Thus the formula:

$$
P_{r}=P_{m} \times\left(\frac{t}{d_{0}}\right)^{\alpha}
$$

and finally after replacing it in the eq. (4) and simplifying we get:

$$
x^{\alpha}+y^{\alpha}+t^{\alpha}<z^{\alpha}
$$

Table 1 shows the solution for $\alpha$ 's natural values from the potential ranges (see section 4.1). Because the value of $t$ is never equal to zero (the reception energy is not negligible), there are intervals of distance $x$ where multi-hop communication is disadvantageous. These ranges are symmetrical about the midpoint of the total transmission distance. So in order to make two-hop routing more energy efficient than a one-hop, the relay should not be too close to either the source or the base station. In the table we also specify the conditions when multi-hop communication is potentially advantageous. If the source-destination distance is too short, the energy saved by relaying does not compensate additional reception cost. This is why the relationship between direct transmission distance $(z)$ and energy consumed by the receiver $(t)$ describes that condition.

In figure 3 the minimum hop transmission distance which guarantees multi-hop routing energy efficiency in a two-hop scheme is plotted. The conclusion depends on the total communication range and reception cost. So for example, if we consider the case of $\alpha=2$, the source distance from the sink 150 meters, and the reception energy corresponding to the 70 meters transmission range $\left(E_{R x}=E_{T x}(70)\right)$, then the two-hop routing is more advantageous than direct communication if the relay is at least 19 meters from both the sink and the source.

The curves have some similarities. There exists a total transmission distance range when direct communication should occur. If the reception cost drops, this interval becomes shorter. Also, for the higher values of $\alpha$ the two-hop routing scheme becomes advantageous earlier (for smaller distances between the sink and the source). If we plot these curves on the same graph, they will be superimposed with the graph of $\alpha=2$ on the top and $\alpha=5$ on the bottom and the others in between. This gives us an idea of how the curves may look for non-integer values of $\alpha$. They will have similar shape and location close to the graph of nearest value of $\alpha$.
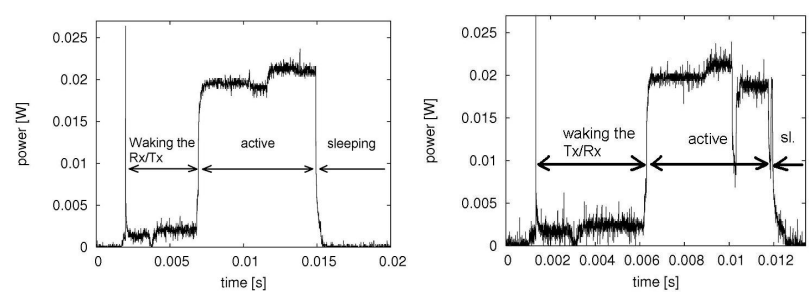

\section{Figure 4. Total power consumption when the node is receiving (a) and sending (b) data.}

\subsection{Total energy drained from the node}

In the previous paragraph we analyzed when the smallest amount of energy is radiated in two-hops and direct communication. We are interested in the energy being drained from the batteries and in this section we find the relationship between these quantities. Figure 4 shows the power consumption when the node is transmitting and receiving data. The relationship between the energy use at the sleeping mode to communication is significant and can differ by up to a hundred times. This is why we can only consider energy drained by the transceiver and neglect other devices. Figure 1 summarizes the relationship between power consumed and that radiated by different transceivers. For all of them we can observe that an increase of the transmitted signal power requires a higher energy supply. If we approximate that correspondence by a linear equation, the conclusions and values obtained in the previous section are still valid. 


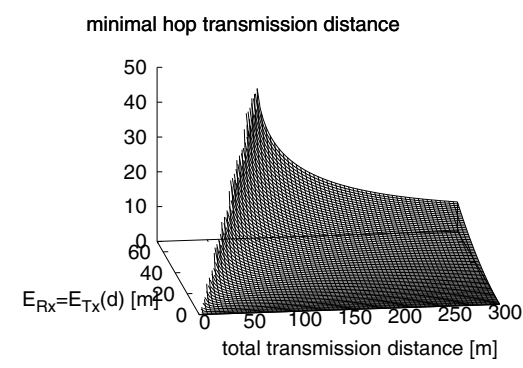

(a)

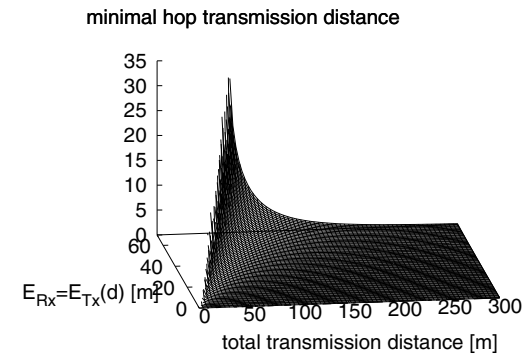

(c)

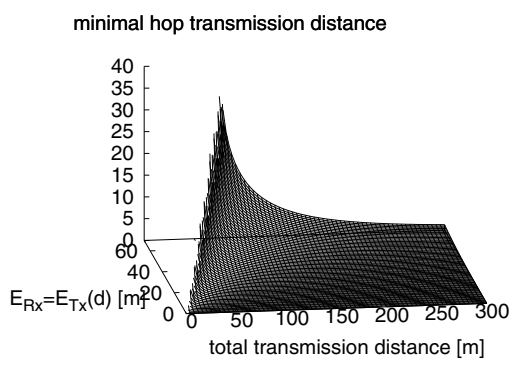

(b)

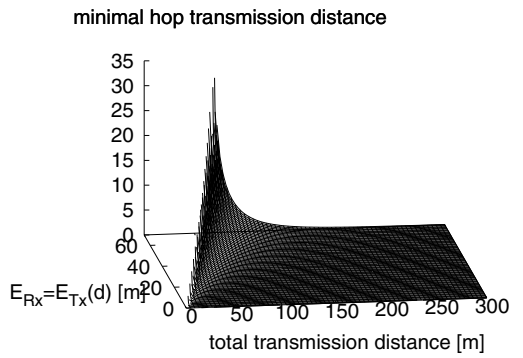

(d)

Figure 3. Minimum hop distance in a two-hop transmission for differen values of path loss: (a): $\alpha=2,(b): \alpha=3$, (c): $\alpha=4$, (d): $\alpha=5$.

\subsection{Two-hop optimal relay location}

Now, when we know the conditions which make multihop routing advantageous over direct transmission (using the MER metric), we investigate the optimal location of the relay to maximize the energy saved by two-hop routing. We start the analysis by expressing the difference between the radiated power in a two-hop system with one using direct communications. With the Friis model and Log-distance path loss model [6] we can represent the power received at the distance $\mathrm{d}$ from the source as:

$$
P_{r}(d)=\beta \times \frac{P_{t}}{d^{\alpha}}
$$

where $\beta$ is a constant specified in the model definition and $P_{t}$ the RF output power. So the power saved by the two-hop transmission is:

$$
P_{\text {saved }}=P_{z}-\left(P_{x}+P_{y}+P_{r}\right)
$$

with $P_{z}, P_{x}$ and $P_{y}$ representing transmission power over distances $z, x, y$ (see figure 2) and $P_{r}$ the reception cost. If we assume nodes send data with the minimum energy to reach the destination and $P_{r}$ is equivalent to communication over distance $\mathrm{t}$ (see section 4.2) the eq. (8) becomes:

$$
P_{\text {saved }}=\frac{P_{m}}{\beta}\left[z^{\alpha}-\left(x^{\alpha}+y^{\alpha}+t^{\alpha}\right)\right]
$$

In order to find the maximum of $P_{\text {saved }}$ we explore eq. (9) for a sample value of $\alpha$. The calculations can be found in the Appendix.

The results show that the inference is the same for all potential values of $\alpha$. The optimal location of a two-hop relay is half way between the source and the base station. We deduce this result from the analysis of the output power of the transceiver. However, as stated in section 4.3, the conclusion is identical for the energy cost. If two-hop communications is more advantageous than direct transmission, it is optimal when the relay is equally distant from the source and the sink.

\section{Experiment results}

In the previous section the analysis is restricted to an ideal chain topology, where nodes are perfectly aligned and propagation noise is neglected. In real scenarios, randomness of the signal can have a significant impact on the received power, and thus the communication scheme energy efficiency. In this section, we perform the experiments with a sensor network to verify the conclusions deduced from the theoretical analysis. The network is composed of 10 nodes which are aligned to simulate the dense networks communication chain. We employed a commonly used sen- 


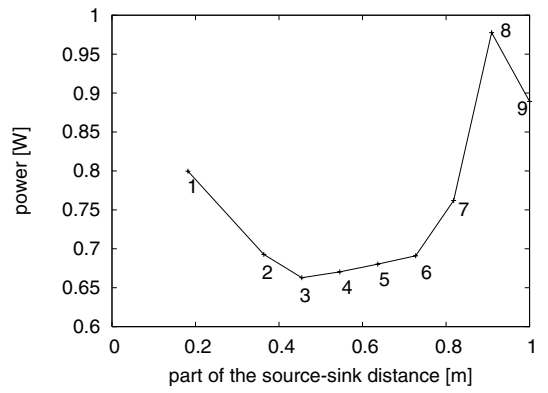

Figure 5. Power consumption by the nodes.

sor network platform: Tmote Sky mote and the networking stack as implemented in TinyOS. Each Tmote sky node has a $2.4 \mathrm{GHz}, 250 \mathrm{kbps}$ IEEE 802.15.4 Chipcon wireless transceiver. Because of its power consumption properties (see figure 1: the maximum transmission power equals 0 $\mathrm{dBm}$ ) we extrapolate the results for a $20 \mathrm{dBm}$ transmitter in the following way. We place the source and the sink at the maximal possible communication distance. In between, we place a line of potential relays. We measure first the path loss coefficient (we obtained $\alpha=2.6$ and $P_{0}=-57 \mathrm{dBm}$, see eq. (1)) when the radiated power is $0 \mathrm{dBm}$. Then we assume that the RF output is $20 \mathrm{dBm}$ and from the measured signal strength at each relay and base station we calculate their extrapolated distance to the source. In the next step we analyze the energy efficiency of both schemes: direct and two-hop transmission. To do that we measure the minimal power radiation to reach the sink from each node location and also to send data from every relay to the base station. From this value we calculate the total power consumption by a linear approximation for the XBee-PRO module (see figure 1). From that we can estimate the total energy consumption of direct transmission and communication through each relay. These values are plotted on figure 5 , where the node with id $=9$ is the base station. The curve should be symmetrical with respect to the $\mathrm{x}=0.5$ axis. The divergence from that stems from the randomness of the signal strength. Indeed, for the communication with the sink, node 4 requires a significant RF output in comparison to nodes 3 and 5 . We can also observe that there are relay locations for which direct communication with the base station is more advantageous (e.g. node 8). From the experiments we conclude that the minimum one-hop distance entailing the energy efficiency of multihopping is $12 \mathrm{~m}$. However the theoretical value for the given topology and environment equals about $13.5 \mathrm{~m}$.

\section{Conclusion}

We have analyzed the problem of hopping distance strategy in WSN. We have shown when multi-hopping is more energy efficient in comparison to a direct transmission. We also determined the optimal location of the relay for the two-hop communication scheme. These inferences are verified experimentally on sensor networking hardware. In our future work we would like to investigate the energy efficiency of the multi-hop chain when communicating nodes are not aligned.

\section{Appendix}

In order to calculate when the two-hop strategy leads to the maximal power saving, we explore eq. (9). We do the analysis for $\alpha=5$. For other cases the reasoning and conclusion are similar. As stated before, the source, the relay and the base station are aligned so eq. (9) becomes

$$
P_{\text {saved }}=\frac{P_{m}}{\beta}\left[z^{5}-\left(x^{5}+(z-x)^{5}+t^{5}\right)\right]
$$

We want to find when this function reaches a maximum

$$
\frac{\partial P_{\text {saved }}}{\partial x}=\frac{P_{m}}{\beta}\left[5 z^{4}-20 z^{3} x+30 z^{2} x^{2}-20 z x^{3}\right]
$$

For $n=x / z$ (n represents the fraction of $\mathrm{x}$ over $\mathrm{z})$ the eq. (11) becomes

$$
\frac{\partial P_{\text {saved }}}{\partial x}=\frac{P_{m}}{\beta} 5 z^{4}\left[\left(n-\frac{1}{2}\right)\left(-4 n^{2}+4 n-2\right)\right]
$$

$\partial P_{\text {saved }} / \partial x=0$ only for $n=1 / 2$ and this derivative changes the value from positive to negative so the function $P_{\text {saved }}$ achieves the maximum value for $n=1 / 2$. So when the relay is at the half distance between source and the sink, the power saved by the two-hop routing is maximal.

\section{References}

[1] Chipcon. CC2431 Location Engine, Application Note AN042, texas instruments literature no: swra095. edition.

[2] CIRRONET. RF Power Options in ZigBee Solutions, cirronet inc. literature edition, 2005.

[3] M. Haenggi. Routing in ad hoc networks: A case for long hops. IEEE Communications Magazine, 2004. Submitted to Series on Ad Hoc and Sensor Networks.

[4] M. Haenggi. Routing in ad hoc networks-a wireless perspective. In BROADNETS 04: Proceedings of the First International Conference on Broadband Networks, pages 652-660, Washington, DC, USA, 2004. IEEE Computer Society.

[5] M. Haenggi. Twelve reasons not to route over many short hops. In IEEE Vehicular Technology Conference (VTC04 Fall), Los Angeles, CA, SEP 2004.

[6] T. S. Rappaport. Wireless Communications: Principles and Practice. Prentice Hall, July 1996.

[7] B. Yin, H. Shi, and Y. Shang. A two-level strategy for topology control in wireless sensor networks. In ICPADS '05: Proceedings of the 11th International Conference on Parallel and Distributed Systems - Workshops (ICPADS'05), pages 358362, Washington, DC, USA, 2005. IEEE Computer Society. 